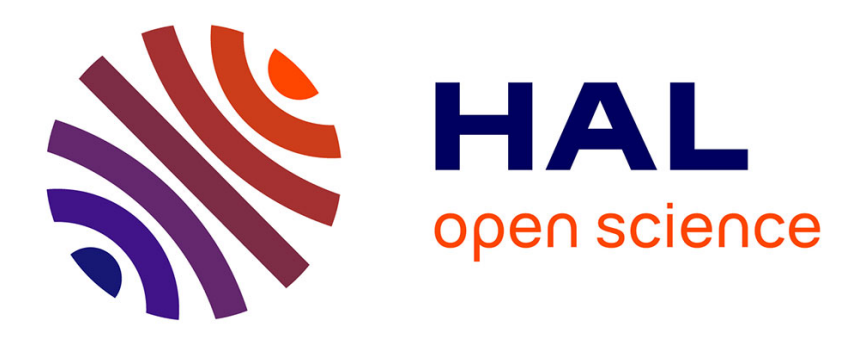

\title{
SIMULATION DE L'ESPACE SONORE SOUS ÉCOUTEURS
}

T. Voinier, F. Briolle

\section{To cite this version:}

T. Voinier, F. Briolle. SIMULATION DE L'ESPACE SONORE SOUS ÉCOUTEURS. Journal de Physique IV Proceedings, 1992, 02 (C1), pp.C1-121-C1-124. 10.1051/jp4:1992123 . jpa-00251194

\section{HAL Id: jpa-00251194 https://hal.science/jpa-00251194}

Submitted on 1 Jan 1992

HAL is a multi-disciplinary open access archive for the deposit and dissemination of scientific research documents, whether they are published or not. The documents may come from teaching and research institutions in France or abroad, or from public or private research centers.
L'archive ouverte pluridisciplinaire HAL, est destinée au dépôt et à la diffusion de documents scientifiques de niveau recherche, publiés ou non, émanant des établissements d'enseignement et de recherche français ou étrangers, des laboratoires publics ou privés. 


\section{SIMULATION DE L'ESPACE SONORE SOUS ECOUTEURS}

\section{T. VOINIER et F. BRIOLLE*}

Equipe de Psychoacoustique, Environnement Acoustique et Perception, Laboratoire de Mécanique et d'Acoustique du CNRS, 31 chemin Joseph-Aiguier, F-13402 Marseille cedex 09, France

*Groupe d'Etudes et de Recherches en Détection Sous-Marines, Chemin de la Gardiole, Le Brusc, F-83140 Six-Fours, France

\section{Abstract}

When listening to a signal through headphones, the sound seems to be inside the head: the action of the head, pinna, shoulders is cancelled. In order to restore natural conditions of listening with headphones, a signal processing technique is achieved.

After a brief review of auditory localization, this paper describes a technique of space simulation under headphones. A prototype based on the real-time convolution of an original monophonic signal with the transfer function of the external ear will be presented. Some examples of applications are suggested.

Dans des conditions naturelles, notre perception de l'espace sonore est possible grâce aux indices présents dans les signaux qui parviennent à nos tympans. Lors de l'écoute sous casque, l'espace sonore est généralement réduit: le son semble être dans la tête, car les indices utile à notre perception de l'espace sont modifiés.

Cet article présente une technique de reconstitution de l'espace sonore subjectif pour le cas de l'écoute au casque.

\section{Principaux indices pour la localisation du son}

En dehors des informations visuelles ou cognitives, nous utilisons les indices présents dans les signaux acoustiques qui arrivent sur les tympans pour identifier la position d'une source sonore.

Les différences de temps et d'intensité entre les signaux qui parviennent aux oreilles de l'auditeur, ainsi que les modifications de leurs spectres sont les indices les plus importants utilisés par le système auditif. De nombreuses études relatives à l'audition binaurale et la localisation du son ont été publiées. On en trouvera une synthèse dans l'ouvrage de Blauert[1].

\section{Différences de niveau}

Les nombreux résultats des mesures des différences de niveau interaurales, effectuées pour une position de la source dans le plan horizontal, sont extrêmement variables. A $4 \mathrm{kHz}$, par exemple, ces différences peuvent atteindre, selon les individus, 15 à $25 \mathrm{~dB}$. En première approximation, cet indice est utilisé par le système auditif aux fréquences supérieures à $1500 \mathrm{~Hz}$. 


\section{Différences de temps}

Dans le plan horizontal les différences de temps, mesurées entre les signaux qui atteignent les deux oreilles, semblent plus stables. Nulles pour des azimuts de $0^{\circ}$ et $180^{\circ}$, elles atteignent environ $0,7 \mathrm{~ms}$, pour des azimuts de $90^{\circ}$ et $270^{\circ}$. Cet indice est utilisé par le système auditif dans toute la gamme des fréquences audibles.

\section{Modifications spectrales}

Deux phénomènes produisent des modifications spectrales:

- La présence du buste dans le champ acoustique créé par une source perturbe celui-ci. Pour certaines positions de la source, la tête joue le rôle d'écran vis à vis d'une des oreilles. Le signal ne peut alors atteindre cette oreille que par diffraction.

- De plus les réflexions du signal sur la tête, les épaules et les pavillons modifient le spectre du signal acoustique qui parvient aux tympans de l'auditeur.

\section{Les fonctions de transfert de l'oreille externe}

On peut donc considérer l'ensemble tête, épaules, pavillons comme deux filtres linéaires dont les caractéristiques reflètent les transformations subies par le signal depuis la source sonore jusqu'aux tympans de l'auditeur. Ces deux filtres rendent compte de tous les indices utiles à la localisation, depuis les différences de temps et d'intensité jusqu'aux modifications spectrales.

Les signaux acoustiques qui arrivent au niveau des tympans de l'auditeur peuvent être mesurés. Un haut-parleur de petite dimension qui émet un signal large bande, est positionné avec précision par rapport au sujet. Des petits microphones situés à proximité des tympans recueillent les pressions acoustiques. Les réponses du haut-parleur et des microphones de mesure doivent être prises en compte pour déterminer les fonctions de transfert de l'ensemble tête, épaules, pavillons, ceci pour chaque oreille et une position donnée de la source. Ces paires de fonctions de transfert sont appellées "Head Related Transfer Functions" (HRTF). Le positionnement précis de la source de bruit par rapport au sujet et l'introduction des microphones dans le conduit auditif rendent ces mesures délicates.

Les modifications dues aux réflexions et à la diffraction variant selon la position de la source dans l'espace, les HRTF seront donc dépendantes de cette position.
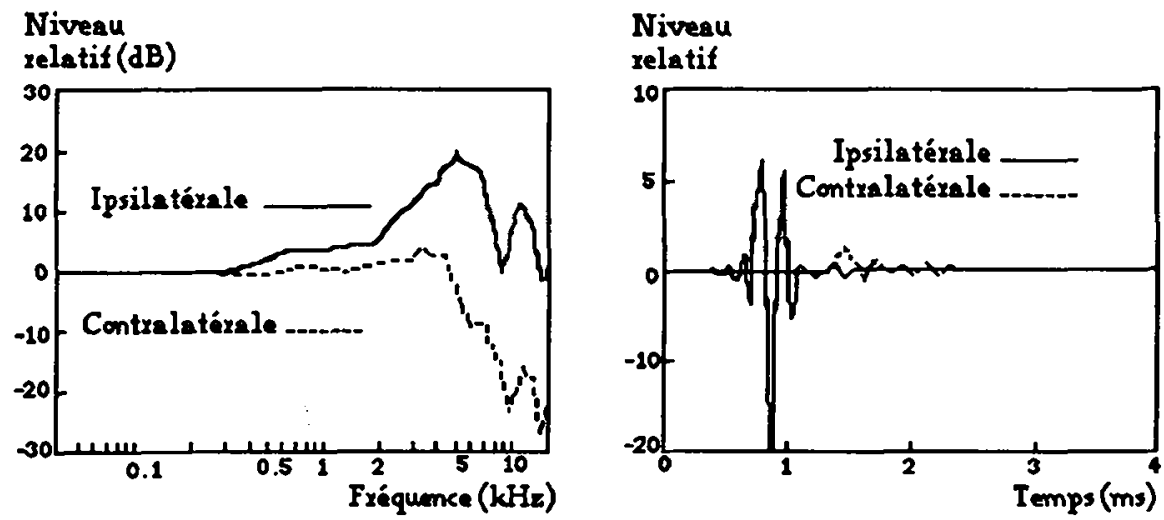

Modules des "Head Related Transfer Functions" et réponses impulsionnelles des oreilles droite et gauche, la source étant placée à $90^{\circ}$ dans le plan horizontal. (Mesure sur tête artificielle NEUMANN KU 81i)

Les modules des HRTF présentés ci-dessus mettent en évidence les différences interaurales d'intensité, ainsi que les modifications spectrales des signaux reçus sur les deux oreilles. Quant aux réponses impulsionnelles (transformées de Fourier inverses des fonctions de transfert), elles font apparaître les différences interaurales de temps et d'intensité. 
Les altérations spectrales que l'on peut observer sont principalement dues aux effets des pavillons. De nombreuses mesures des HRTF (Morimoto et al. [2], Pösselt et al. [3], Schmitz et al. [4], et Wightman et al. [5] ...) ont montré de grandes disparités inter-individuelles, essentiellement dues aux différences anatomiques des pavillons.

\section{Simulation de l'espace sonore sous écouteur}

Lors de l'écoute au casque, le son semble être dans la tête. Les modifications des signaux naturellement réalisées par la tête, les épaules et les pavillons sont annulées avec un casque d'écoute: le signal qui arrive aux tympans de l'auditeur est simplement filtré par le casque. Recréer l'espace sonore subjectif suppose donc de rétablir les conditions naturelles d'écoute, c'est-à-dire rétablir les filtrages évoqués ci-dessus, ce qui peut être obtenu en ajoutant un filtre avant chaque écouteur.

Le calcul de ces filtres est fait en plusieurs étapes: on mesure tout d'abord les HRTF en champ libre, pour une position donnée de la source, puis celle du casque d'écoute utilisé. Les fonctions de transfert des filtres nécessaires à la simulation de l'espace sonore seront le rapport des HRTF à la fonction de transfert du casque d'écoute.

\section{Application}

Nous avons souhaité réaliser un prototype fonctionnant en temps réel, susceptible de donner une impression de mouvement du son dans le plan horizontal.

Puisque nous avons facilement accès à la réponse impulsionnelle des filtres nécessaires à cette simulation, il est commode d'effectuer le filtrage au moyen de filtres numériques à réponse impulsionnelle finie (RIF). L'opération à effectuer est une convolution du signal d'entrée par la réponse impulsionnelle des filtres.

$$
y_{n}=\sum_{k=1}^{m} x_{n-k} \cdot c_{k}
$$

Chaque échantillon, $\mathrm{y}_{\mathrm{n}}$, à la sortie du filtre est formé par la somme des produits des $\mathrm{m}$ derniers échantillons d'entrée, $x_{n-k}$, par les $m$ coefficients $d u$ filtre, $c_{k}$. Cette opération doit être réalisée pour chaque arrivée d'un nouvel échantillon. On dispose donc de l'intervalle de temps séparant l'arrivée de deux échantillons pour effectuer ce calcul; on ne pourra donc traiter qu'un nombre limité de coefficients.

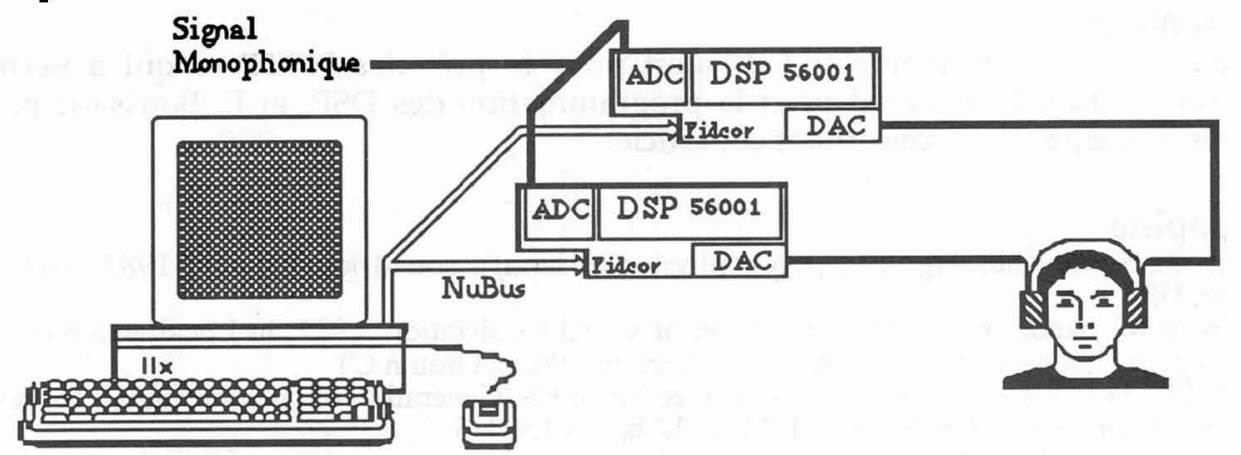

Simulation de l'espace sonore subjectif au moyen de processeurs spécialisés.

L'impression de mouvement suppose de pouvoir changer les réponses des filtres, c'est-àdire leurs coefficients. On doit donc disposer de mesures des fonctions de transfert pour la trajectoire à simuler. Puisque nous voulons reproduire un mouvement dans le plan horizontal, nous avons choisi de mesurer les HRTF tous les $5^{\circ}$ (de $0^{\circ}$ a $360^{\circ}$ ) pour avoir un bon échantillonnage de cette trajectoire. 
L'impression de mouvement est réalisée par changement des réponses des filtres. L'impression de déplacement continu, due au faible pas d'échantillonnage spatial, est encore améliorée par l'utilisation d'une interpolation entre deux réponses impulsionnelles.

Le filtrage est réalisé par deux processeurs spécialisés (DSP 56001), ce qui permet d'utiliser en temps réel, à la fréquence d'échantillonnage de $44.1 \mathrm{kHz}$, des filtres de 256 coefficients. Ces processeurs sont pilotés par un ordinateur hôte (Macintosh IIx), qui permet la commutation des filtres en moins de $30 \mathrm{~ms}$, ce qui contribue à l'impression de déplacement continu de la source dans l'espace sonore.

\section{Démonstration}

Une démonstration des possibilités de ce prototype sera proposée. Un magnétophone numérique RDAT et un lecteur de disques numériques seront utilisés comme source de signaux. Nous proposerons des restitutions de l'espace sonore en temps réel par simulation des indices utiles à la localisation:

- différences d'intensité, différences de temps d'arrivée, et combinaison de ces deux indices.

- différences d'intensité, de temps d'arrivée et modifications spectrales. La technique décrite ci-dessus sera utilisée. La qualité de simulation pourra être évaluée en écoutant un espace sonore simulé au moyen de HRTF mesurées sur tête artificielle, et non sur leurs propres oreilles.

\section{Conclusion}

De nombreuses applications peuvent être réalisées. On peut imaginer l'intégration de filtres dans des appareils audionumériques grand public (balladeurs, platines laser...) qui donneraient à l'auditeur une sensation d'espace sonore lors de l'écoute sous casque. Le développement des enregistrements numériques permet aussi d'envisager une composition musicale destinée à être écoutée sous casque: le compositeur aurait la possibilité d'utiliser l'espace sonore et en particulier des illusions de mouvement du son dans l'espace.

Des applications spécifiques sont en cours de développement, comme par exemple une console de mixage "binaurale" [6], ou un système d'aide aux opérateurs de détection sousmarine [7] ou encore un système de guidage des cosmonautes dans l'espace [8].

\section{Remerciements}

Les auteurs tiennent à remercier J. Blauert pour le prêt des HRTF ce qui a permis de commencer ce travail, A. Freed pour la programmation des DSP, et P. Boussard pour ses remarques et suggestions concernant cet article.

\section{Bibliographie}

[1] Blauert J., Spatial hearing: The psychophysics of human sound localization, 1983, MIT Press, Cambridge, USA

[2] Morimoto M., Ando Y., On the simulation of sound localization, 1982, in Localization of sound: Theory and applications, Gathehouse R.W. ed, Amphora Press, Groton CT

[3] Pösselt Ch., Schröter J., Opitz M., Divenyi P.\& Blauert J., Generation of binaural signals for research and home entertainment, 1986, Proc. of I. C. A. 12th, vol 1, B1-6

[4] Schmitz A., Vorländer M., Messung von Aubenohrstobantworten mit Maximalfolgen-HadamardTranformation und deren Anwendung bei Inversionsversuchen, 1990, Acustica, vol. 71, 257-268

[5] Wightman F.L., Kistler D.J., Perkins M.E., A new approach to the study of human localization., 1987 , in Directional hearing, Yost \& Gourevitch Eds, Springer Verlag, 26-48

[6] Persterer A., A very high performance digital audio processing system, 1979, J.A.E.S. vol $27 \mathrm{~N}^{\circ} 12$

[7] Briolle F., Detection and classification of the audiophonic sonar signal: perspectives of space simulation under headphones, Undersea Defense Technology, Avril 91

[8] Wenzel E.M., Wightman F.L., Kistler D.J., Foster S.H., The convolvotron: Realtime Synthesis of out-of-head localization, 1988, Joint Meeting of the Acoustical Society of America and the Acoustical Society of Japan, Honolulu, November 14-18 